\title{
Was Sie als Ärztin oder Arzt über die Weiterbildung unbedingt wissen müssen
}

\author{
Ch. Hänggeli, Geschäftsleiter Aus-, Weiter- und Fortbildung (AWF)
}

\section{Wer hilft mir bei Fragen im Bereich der ärztlichen Weiterbildung weiter?}

Das Sekretariat Aus-, Weiter- und Fortbildung (AWF) der FMH ist Anlaufstelle für alle Fragen im Bereich der ärztlichen Weiter- und Fortbildung. Sämtliche wichtigen Informationen finden Sie aufunserer Website (www.fmh.ch/awf). Wir stehen Ihnen zu Bürozeiten, aber auch gerne persönlich zur Verfügung (Elfenstrasse 18, Postfach 293, 3000 Bern 16, Tel. 03135911 11, Fax 03135911 12, E-Mail: diplome@hin.ch).

Bitte beachten Sie auf unserer Website die «Wegleitung FMH/BAG», welche im Hinblick auf die Bilateralen Verträge mit der EU Auskunft gibt über

- den Erwerb von Ausbildungsdiplomen, Weiterbildungstiteln und Fortbildungszertifikaten;

- die Anerkennung ausländischer Ausbildungsdiplome und Weiterbildungstitel;

- die Zulassung zum Arztberuf in gesundheitspolizeilicher, sozialversicherungsrechtlicher und ausländerrechtlicher Hinsicht.

\section{Welche Anforderungen muss ich für einen bestimmten Weiterbildungstitel erfüllen?}

Die allgemeinen und gemeinsamen Grundsätze der ärztlichen Weiterbildung sind in der Weiterbildungsordnung (WBO) geregelt. Daneben existieren 44 Weiterbildungsprogramme für jeden einzelnen Facharzttitel und den Titel «Praktischer Arzt/Praktische Ärztin». Bitte nehmen Sie sich Zeit, das von Ihnen gewählte Weiterbildungsprogramm zu studieren, damit Sie über die spezifischen Anforderungen Ihres Curriculums informiert sind (allfällige Reihenfolge und Kategorien der zu absolvierenden Weiterbildungsjahre, Besuch von Kursen, Erstellung von Publikationen, etc.). Die Liste der anerkannten Weiterbildungsstätten gibt darüber Auskunft, an welchen Institutionen die Weiterbildung absolviert werden kann und bis zu welcher Dauer sie angerechnet wird. Tätigkeiten an Spitälern, Kliniken und Arztpraxen, die nicht auf der Liste aufgeführt sind, werden Ihnen nicht angerechnet! Stellen Sie bei der Anstellung sicher, dass Ihnen ein schriftlicher Arbeitsvertrag ausgehändigt wird und es sich um eine Weiterbildungsstelle handelt, für die Ihnen ein offizielles FMH-Zeugnis ausgestellt wird.

\section{Kann ich mich auch im Ausland weiterbilden?}

Holen Sie vor dem Antritt einer Stelle im Ausland immer zuerst die Zustimmung der Titelkommission ein, damit Sie mit der Anrechenbarkeit des Auslandaufenthaltes keine Schwierigkeiten erhalten. Grundsätzlich wird die Tätigkeit an gleichwertigen Weiterbildungsstätten im Ausland als Anteil der reglementarischen Weiterbildung anerkannt. Mindestens die Hälfte der fachspezifischen Weiterbildung muss allerdings an anerkannten Weiterbildungsstätten in der Schweiz absolviert werden. Beachten Sie allfällige spezielle Vorschriften im Weiterbildungsprogramm über die Definition der fachspezifischen Weiterbildung, die Kategorie der Weiterbildungsstätten und die Anrechenbarkeit von Operationen.

Benutzen Sie bitte für alle Anfragen immer das offizielle elektronische Gesuchsformular auf unserer Website www.fmh.ch/awf.

\section{Kann ich meine Weiterbildung auch in Teilzeit absolvieren?}

Ja, unter folgenden Bedingungen: Anrechenbar sind nur Arbeitspensen von mindestens 50\%. Wenn sich im Weiterbildungsprogramm keine spezielle Regelung findet, gilt im übrigen folgendes:

Höchstens die Hälfte der fachspezifischen Weiterbildung kann in Teilzeit absolviert werden. Nicht-fachspezifische Weiterbildung können Sie gänzlich in Teilzeit leisten.

Viele Weiterbildungsprogramme lassen es bereits zu, dass die ganze Weiterbildung in Teilzeit absolviert werden kann. 
Gibt es eine Mindestdauer für die Anrechnung einer Weiterbildungsperiode?

Ja! Damit eine Weiterbildungsperiode angerechnet werden kann, muss eine Mindestdauer von 6 Monaten an der gleichen Weiterbildungsstätte ausgewiesen werden (bei Teilzeit: Verlängerung pro rata). Ausnahmsweise sind pro Facharzttitel maximal 3 Kurzperioden von mindestens 3 Monaten zugelassen (für einen Schwerpunkt ist 1 Kurzperiode anrechenbar). Praxisassistenz und Militärdienst sind bereits ab einer ununterbrochenen Dauer von 1 Monat anrechenbar und zählen nicht als Kurzperiode.

\section{Wird mir eine Volontärtätigkeit angerechnet?}

Volontärtätigkeit wird Ihnen für die Dauer von 3 Monaten angerechnet und zählt in jedem Fall als Kurzperiode.

\section{Wird mir meine Tätigkeit als Praxisassistent oder Stellvertreter angerechnet?}

Praxisassistenz und Stellvertretung werden Ihnen nur und soweit angerechnet, als es im entsprechenden Weiterbildungsprogramm vorgesehen ist. Im übrigen gilt Art. 34 der Weiterbildungsordnung.

\section{Muss ich das FMH-Zeugnis jedes Jahr der FMH einschicken?}

Nein! Senden Sie uns bitte keine Zeugnisse, bis Sie sämtliche im Weiterbildungsprogramm geforderten Belege vollständig gesammelt haben. Jede Weiterbildungsperiode muss mit einem anrechenbaren FMH-Zeugnis und allfälligen fachspezifischen Zusatzblättern bestätigt sein. Der Weiterbildungsverantwortliche führt mindestens jedes Jahr ein Evaluationsgespräch, dessen Ergebnisse in den offiziellen Protokollblättern festzuhalten sind.

\section{Kann ich die Weiterbildungsstätte auch bewerten?}

Die FMH führt jedes Jahr eine Umfrage bei allen Assistentinnen und Assistenten durch, in der die Weiterbildungsstätte einer detaillierten Beurtei- lung unterzogen wird. Beteiligen Sie sich aktiv an dieser Umfrage, damit allfällige Mängel in der Weiterbildung behoben werden können.

\section{Wie muss ich mein Titelgesuch einreichen?}

Der Antrag zur Erteilung eines Facharzttitels ist ausschliesslich und direkt auf der Website der FMH (www.fmh.ch/awf) mit dem elektronischen Gesuchsformular zuhanden der Titelkommission einzureichen. Prüfen Sie am Schluss der Eingabe anhand der Checkliste genau, ob Sie sämtliche im Weiterbildungsprogramm geforderten Unterlagen zusammengestellt haben. Reichen Sie ausschliesslich gut lesbare Fotokopien aller geforderten FMH-Zeugnisse inkl. allfälliger fachspezifischer Zusatzblätter und der dazugehörigen Evaluationsprotokolle ein. Unvollständige Dossiers und Originaldokumente müssen wir leider retournieren! FMH-Zeugnisse können nur validiert werden, wenn sie sowohl Ihre Unterschrift wie auch diejenige des autorisierten Leiters der anerkannten Weiterbildungsstätte enthalten.

Wenn Sie keine Möglichkeit haben, das offizielle Gesuchsformular auf unserer Website auszufüllen, helfen wir Ihnen gerne weiter. In diesem Fall müssen Sie mit einer längeren Gesuchsbearbeitung rechnen.

\section{Wann ändert sich ein Weiterbildungs- programm?}

Die Halbwertszeit in der Medizin ist sehr kurz. Dementsprechend ändern die Anforderungen der einzelnen Weiterbildungsprogramme relativ rasch. Kontrollieren Sie regelmässig, ob das von Ihnen gewählte Curriculum aktualisiert worden ist (www.fmh.ch/awf). Neue Anforderungen werden in aller Regel mit einer Übergangszeit von 3 Jahren eingeführt. Das heisst, wer innert 3 Jahren seit der Inkraftsetzung des neuen Programmes die Weiterbildung beendet, kann nach altem Programm abschliessen. Andernfalls sind die Bedingungen des neuen Weiterbildungsprogrammes zu erfüllen.

\section{Muss ich die Facharztprüfung bestehen?}

Alle Fachgesellschaften führen inzwischen eine Facharztprüfung durch. Ob Sie an der Facharztprüfung nur teilnehmen oder ob Sie sie bestehen müssen, hängt vom jeweiligen Fachgebiet ab. 
Bitte beachten Sie die einschlägigen Publikationen in der Schweizerischen Ärztezeitung und auf unserer Website. Die Termine für die Facharztprüfung und die Anmeldemodalitäten werden jeweils 6 Monate vorher in der Schweizerischen Ärztezeitung publiziert.

Muss ich zum Erwerb eines Facharzttitels, Schwerpunktes, Fähigkeits- oder Fertigkeitsausweises Mitglied der FMH sein?

Für sämtliche eidgenössischen Weiterbildungstitel (= alle Facharzttitel und praktischer Arzt/ praktische Ärztin) ist die Mitgliedschaft bei der FMH nicht obligatorisch. Anders verhält es sich mit den Schwerpunkten, Fähigkeits- und Fertigkeitsausweisen, welche nicht dem Bundesgesetz über die Freizügigkeit der Medizinalberufe (FMPG) unterstehen und von der FMH im Sinne einer Dienstleistung exklusiv an ihre Mitglieder abgegeben werden. Auch die Mitgliedschaft bei einer Fachgesellschaft ist freiwillig. Bitte erkundigen Sie sich nach den Vorteilen, die mit einer Mitgliedschaft verbunden sind.

\section{Kann ich eine Weiterbildungsperiode gleichzeitig für $z$ wei verschiedene Facharzttitel anrechnen lassen?}

Ja Sie können! Zwei Jahre Innere Medizin sind beispielsweise gleichzeitig für die Facharzttitel Innere Medizin, Allgemeinmedizin und Kardiologie anrechenbar. Der Erwerb mehrerer Facharzttitel wird damit massiv erleichtert.

\section{Muss ich als Mitglied der FMH Gebühren bezahlen?}

Bitte beachten Sie die Gebührenordnung auf unserer Website (www.fmh.ch/awf). Als Mitglied der FMH profitieren Sie von folgenden GratisDienstleistungen:

- Auskünfte und Beratungen in allen Weiterbildungsfragen (z.B. Anrechnung ausländischer Weiterbildung);

- Erstellung eines Weiterbildungsplanes, anhand dessen die Titelkommission Ihre bisherige Weiterbildung validiert und Sie auf die noch fehlenden Weiterbildungsanforderungen hinweist. 